\title{
FSZ-groups and Frobenius-Schur indicators of quantum doubles
}

\author{
M. Iovanov, G. Mason and S. Montgomery
}

\begin{abstract}
We study the higher Frobenius-Schur indicators of the representations of the Drinfel'd double of a finite group $G$, in particular the question as to when all the indicators are integers. This turns out to be an interesting group-theoretic question. We show that many groups have this property, such as alternating and symmetric groups, $P S L_{2}(q), M_{11}, M_{12}$ and regular nilpotent groups. However, we show there is an irregular nilpotent group of order $5^{6}$ with non-integer indicators.
\end{abstract}

\section{Introduction}

In the representation theory of finite groups, Frobenius-Schur indicators and their higher analogs are an important set of invariants. For Hopf algebras, the second indicator was generalized in $[\mathrm{LM}]$, and higher indicators in [KSZ]. They were then extended to quasi-Hopf algebras [MN05] and tensor categories [NS07, NS11]. An important property is that they are gauge invariants, that is, invariants of the tensor category of representations. They proved to have many interesting applications; for example, in [KSZ] it is proved that for $H$ a semi-simple Hopf algebra over $\mathbb{C}$, the exponent of $H$ and the dimension of $H$ have the same prime factors, answering a question of [EG]. They can also be used to distinguish between the tensor categories having the same Grothendieck ring, such as the 8 types of non-isomorphic semi-simple quasi-Hopf algebras (tensor categories) of dimension 8 over $\mathbb{C}$. Thus knowing the possible values of the indicators is an interesting problem. In this paper, we study the case of $H=D(G)$, the Drinfel'd double of a finite group $G$.

Throughout we work over the complex numbers $\mathbb{C}$. We refer to $[\mathrm{M}]$ for basic facts about Hopf algebras. Let $H$ be a semi-simple Hopf algebra over $\mathbb{C}$

1991 Mathematics Subject Classification. MSC Primary 20D99, 20C99; Secondary $16 \mathrm{~T} 05$. 
and let $V$ be an irreducible representation of $H$ with character $\chi$. As defined in [KSZ], the $n$th Frobenius-Schur indicator of $V$ is given by

$$
\nu_{n}(V):=\chi\left(\Lambda^{[n]}\right),
$$

where $n$ is a positive integer, $\Lambda$ is a normalized integral of $H$ and for any $h \in H, h^{[m]}$ is the $n$th Hopf power of $h$. That is, $h^{[m]}=\left(\mu^{n-1} \circ \Delta_{n-1}\right)(h)=$ $\sum_{h} h_{1} h_{2} \ldots h_{m}$, where $\Delta_{n-1}$ is the $(n-1)$ st iteration of the comultiplication $\Delta$ of $H$ and $\mu$ is multiplication.

In the classical case of a group algebra, $H=\mathbb{C} G$ for a finite group $G$, $\nu_{n}(V):=\chi\left(|G|^{-1} \sum_{g \in G} g^{n}\right)$, and it is known (see (2.6) below) that all higher indicators are integers.

For arbitrary Hopf algebras this is not the case. Let $e=e(H)$ denote the exponent of $H$ : this is the smallest number $m$ such that $h^{[m]}=\varepsilon(h) 1$ for all $h \in H$, and always exists if $H$ is semi-simple. Kashina et al. [KSZ, Section 3.2] show that $\nu_{n}(V)$ is an algebraic integer in $\mathbb{Q}\left(\zeta_{e}\right)$, the eth cyclotomic field, and moreover $\nu_{n}(V) \in \mathbb{Q}\left(\zeta_{e / \operatorname{gcd}(n, e)}\right) \cap \mathbb{Q}\left(\zeta_{n}\right)$. They construct an example in which the value is not a rational integer.

In this paper, we are concerned with the Hopf algebra $D(G)$, the Drinfel'd double of $G$. It was an open question whether or not all indicator values for $D(G)$ are integers.

Definition 1.1. For a positive integer $n$, we say that $G$ is an $F S Z_{n}$-group if every $n$th indicator $\nu_{n}(V)$ for $D(G)$ is an integer. We say that $G$ is an $F S Z$-group if it is $F S Z_{n}$ for all $n$.

Thus the question amounts to this: is every finite group $F S Z_{n}$ for all $n$ ? We will show that a variety of finite groups indeed have this property. These include symmetric and alternating groups, 2-dimensional linear groups $P S L_{2}(q)$, the sporadic simple groups $M_{11}$ and $M_{12}$, regular $C N$ groups (the centralizer of each non-identity element is a direct product of regular $p$-groups in the sense of $\mathrm{P}$. Hall $[\mathrm{H}]$ ), and groups whose exponent is "not too composite". In a slightly different direction, we show that every $G$ is $F S Z_{n}$ for $n=2,3,4$ or 6 (in fact Linchenko and Montgomery [LM] show $\left.\nu_{2}(V) \in\{0, \pm 1\}\right)$ ), and that if $G$ is not $F S Z_{n}$ then there is an element $g \in G$ of order 5 or greater than 7 such that $C_{G}(g)$ is not $F S Z_{n}$. These results suggest that the class of $F S Z$-groups is extensive. Indeed, we have made no attempt to be as general as possible, and there are certainly other classes of finite groups which can be shown to be $F S Z$ using the methods that we develop in the present paper. The most tantalizing open question is whether every finite simple group is $F S Z$. 
Despite the proliferation of $F S Z$-groups, it turns out that there are indeed non- $F S Z$-groups, indeed there are infinitely many of them. Because of the results mentioned in the previous paragraph, a natural first place to look for a non-FSZ group would be an irregular 5-group of order $5^{6}$ and exponent $5^{2}$, and we shall see that indeed there are non- $F S Z$-groups of this type.

The paper is arranged as follows. In Section 2 we give the basic definitions and some results about the indicators of $D(G)$ of a combinatorial and character-theoretic nature. In Section 3, we use these results to give (Theorem 3.1) various characterizations of $F S Z$-groups. We also introduce $F S Z^{+}$-groups; these are finite groups satisfying a natural group-theoretic condition that is qualitatively stronger than the $F S Z$ condition. $F S Z^{+}$ groups have a very convenient inductive property (Theorem 3.8) that we use extensively in Sections 4 and 5 to show that various classes of groups are $F S Z^{+}$. Indeed, we know of no group that is $F S Z$ but not $F S Z^{+}$. In Section 6 we show that all symmetric and alternating groups are $F S Z$. Section 7 is devoted to counterexamples, i.e., the existence of non- $F S Z$-groups, including dedicated computer programs that can run on GAP to test the $F S Z$ property.

Etingof's result, that wreathed products $S_{n} \prec A$ ( $A$ abelian) are $F S Z$, is discussed in Section 6. It has now appeared [E].

\section{The sets $G_{n}(u, g)$}

From now on, $G$ will always denote a finite group. Let $I(G)$ denote the irreducible characters of $G, R(G)$ the ring of virtual characters of $G(\mathbb{Z}$ linear combinations of irreducible characters), $\operatorname{cf}(G)$ the $\mathbb{C}$-algebra of class functions of $G$, and $\langle,\rangle_{G}$ the usual inner product defined on $c f(G)$. We also let $\mathbb{C}^{G}$ denote the Hopf algebra of functions on $G$.

Fix a conjugacy class $\mathcal{C}$ of $G$ and an element $u \in \mathcal{C}$. Let $C(u)$ be the centralizer of $u$ in $G$, and let $W$ be an irreducible representation of $C(u)$. Then with a suitable action of $\mathbb{C}^{G}, V=\mathbb{C} G \otimes_{\mathbb{C} C(u)} W$ is an irreducible representation of $D(G)$; moreover all irreducible representations of $D(G)$ are obtained in this way $[\mathrm{KMM}]$.

Let $\eta$ denote the character of $C(u)$ furnished by $W$, and $\chi=\chi_{\eta}$ denote the corresponding $D(G)$-character of $V$. Given this data, we use the notation $V=V_{C(u), \eta}$ and $W=W_{\eta}$.

For $g \in G$, define (as in [KSZ, 7.2])

$$
G_{n}(u, g)=\left\{a \in G \mid\left(a u^{-1}\right)^{n}=a^{n}=g\right\}
$$


with

$$
G_{n}(u)=\bigcup_{g \in G} G_{n}(u, g)=\left\{a \in G \mid\left(a u^{-1}\right)^{n}=a^{n}\right\} .
$$

For a subgroup $H \subseteq G$ we also set

$$
G_{n}^{H}(u, g)=\left\{a \in H \mid\left(a u^{-1}\right)^{n}=a^{n}=g\right\} .
$$

It is easy to see that if $a \in G_{n}(u)$, then $a^{n} \in C(u)$. Thus $G_{n}(u, g)=\phi$ unless $g \in C(u), G_{n}(u, g)=G_{n}^{C(g)}(u, g)$, and

$$
G_{n}(u)=\bigcup_{g \in C(u)} G_{n}(u, g)
$$

Combining the formula for $\nu_{n}(V)$ given in $[\mathrm{KSZ}, 7.4]$ with our remarks about $G_{n}(u)$, we see

$$
\begin{aligned}
\nu_{n}\left(\chi_{\eta}\right) & =|C(u)|^{-1} \sum_{g \in C(u)}\left|G_{n}(u, g)\right| \eta(g) \\
& =|C(u)|^{-1} \sum_{a \in G_{n}(u)} \eta\left(a^{n}\right)
\end{aligned}
$$

The following character-theoretic characterization of the $n$th indicator will be useful. First we introduce

$$
\zeta_{n}^{C(u)}=\zeta_{n}: C(u) \rightarrow \mathbb{Z}, g \mapsto\left|G_{n}(u, g)\right|
$$

Note that $\zeta_{n} \in c f(C(u))$.

Proposition 2.3. The following hold:

$$
\begin{aligned}
\zeta_{n} & =\sum_{\eta \in I(C(u))} \nu_{n}\left(\chi_{\eta}\right) \eta \\
\nu_{n}\left(\chi_{\eta}\right) & =\left\langle\zeta_{n}, \eta\right\rangle_{C(u)}
\end{aligned}
$$

Proof. Because $\zeta_{n}$ is a class function, there is a decomposition $\zeta_{n}=$ $\sum_{\eta \in I(C(c))} \alpha_{n}(\eta) \eta$, for scalars $\alpha_{n}(\eta)$. Since $\alpha_{n}(\eta)=\left\langle\zeta_{n}, \eta\right\rangle_{C(u)}=|C(u)|^{-1}$ $\sum_{g \in C}\left|G_{n}(u, g)\right| \eta(g)$, we must have $\alpha_{n}(\eta)=\nu_{n}\left(\chi_{\eta}\right)$ by $(2.1)$, whence $(2.4)$ and (2.5) follow.

It will be worthwhile to make explicit the special case when $u=1$, corresponding to the original $n$th indicators $\nu^{G}(\eta)$ of the irreducible characters 
$\eta$ of $G$. (Here, $\eta$ is also an irreducible character for $D(G)$ and coincides with $\left.\chi_{\eta}\right)$. In this case we write $\varphi_{n}^{G}$ in place of $\zeta_{n}$. Then

$$
\varphi_{n}^{G}=\sum_{\eta \in I(G)} \nu_{n}^{G}(\eta) \eta
$$

and it is well known that $\varphi_{n}^{G} \in R(G)$. Indeed by $(2.2), \nu_{n}^{G}(\eta)=\left\langle 1, \lambda^{n}(\eta)\right\rangle_{G}$, where 1 is the trivial 1-dimensional character and $\lambda^{n}(\eta)$ is the virtual character given by the $n$th Adams operator applied to $\eta$ (see [CR, Section 12.B] for Adams operators).

Lemma 2.7. There is a bijection of sets

$$
G_{n}(u, g) \rightarrow G_{n}\left(u, g^{-1}\right), \quad a \mapsto u a^{-1} .
$$

Proof. This is straightforward to check. The inverse map is $b \mapsto b^{-1} u$.

Remark 2.8. The indicators of all representations of $D(G)$ are real.

Indeed, apply complex conjugation to (2.1). Since $\overline{\eta(y)}=\eta\left(y^{-1}\right)$, we see $\overline{\nu_{n}\left(\chi_{\eta}\right)}=\nu_{n}\left(\chi_{\eta}\right) \in \mathbb{R}$. (S-H. Ng has shown that the indicators of the double $D(H)$ for any $H$ are real $[\mathrm{N}]$; our special case is much easier to prove.)

We also give a simpler proof for $D(G)$ of the result of [KSZ, Section 2.3 ] that $\nu_{n}(V)$ is an algebraic integer in $\mathbb{Q}\left(\zeta_{e} / \operatorname{gcd}(n, e)\right)$. It will follow that to determine whether $G$ is an $F S Z$-group, it suffices to consider $\nu_{d}(V)$ for $d \mid e$.

Lemma 2.9. Let $e$ be the exponent of $G, n$ a positive integer and $d=$ $\operatorname{gcd}(n, e)$. For any irreducible representation $V$ of $D(G), \nu_{d}(V)$ and $\nu_{n}(V)$ are integers in $\mathbb{Q}\left(\zeta_{e / d}\right)$ and there is $\sigma \in \operatorname{Gal}\left(\mathbb{Q}\left(\zeta_{e / d}\right) / \mathbb{Q}\right)$ depending only on $n$ such that $\nu_{n}(V)=\sigma\left(\nu_{d}(V)\right)$.

In particular, to show all indicators of $V$ are rational integers it is enough to show that $\nu_{d}(V) \in \mathbb{Z}$ for all $d \mid e$.

Proof. We use the formula in equation (2.2) for the indicators of a general irreducible representation of $D(G)$. Let $d=\alpha n+\beta e$; we note that $h^{n}=$ $\left(h u^{-1}\right)^{n} \quad$ implies $\quad h^{d}=h^{n \alpha+e \beta}=\left(h^{n}\right)^{\alpha}=\left(h u^{-1}\right)^{n \alpha}=\left(h u^{-1}\right)^{n \alpha+e \beta}=$ $\left(h u^{-1}\right)^{d}$. Hence $G_{n}(u)=G_{d}(u)$. So $\sum_{h \in G_{n}(u)} \eta\left(h^{n}\right)=\sum_{h \in G_{d}(u)} \eta\left(\left(h^{d}\right)^{q}\right)$, where $n=d q$; moreover, $h^{d}=\left(h^{n}\right)^{\alpha} \in C(u)$. Since $\left(h^{d}\right)^{e / d}=1$, we see that $\eta\left(h^{d}\right)$ is an algebraic integer in $\mathbb{Q}\left(\zeta_{e} / d\right)$, since it is the trace of a matrix of order $e / d$. Since $\operatorname{gcd}(e / d, q)=1, \sigma=\left\{t \mapsto t^{q}\right\} \in \operatorname{Gal}\left(\mathbb{Q}\left(\zeta_{e / d}\right) / \mathbb{Q}\right)$, and we obviously have $\sigma\left(\eta\left(h^{d}\right)\right)=\eta\left(h^{n}\right)$. Therefore, $\sigma\left(\nu_{d}(V)\right)=\nu_{n}(V)$. 
This lemma was used in [C] and is used in some of our computations in Section 7.

\section{Characterizations of $F S Z$-groups}

In this section, we give various characterizations of $F S Z$-groups. We also introduce the idea of $F S Z^{+}$-groups; these are special kinds of $F S Z$-groups that are very natural and are better behaved from a group-theoretic perspective (Theorem 3.8). It will turn out that for most of the examples considered in the following sections, it is the stronger $F S Z^{+}$property that we will establish. Indeed, we do not know of a group that is $F S Z$ but not $F S Z^{+}$.

We begin with some character-theoretic characterizations. We fix some notation which is standard. Let $H$ be a subgroup of $C(u)$. If $\eta$ is a character of $C(u)$, then $\operatorname{Res}_{H}^{C(u)}(\eta)$ is the restriction of $\eta$ to $H$; conversely for $\lambda$ a character of $H, \operatorname{Ind}_{H}^{C(u)}(\lambda)$ denotes the induced character from $H$ to $C(u)$. We use $\zeta_{n}$ as in Proposition 2.3. The rational conjugacy class of $g \in C(u)$ consists of all $C(u)$-conjugates of $g^{m}$ for all integers $m$ coprime to $|C(u)|$.

Theorem 3.1. Let $n$ be a positive integer. The following are equivalent:

(1) $\nu_{n}\left(\chi_{\eta}\right) \in \mathbb{Z}$ for each $\eta \in I(C(u))$;

(2) $\zeta_{n} \in R(C(u))$;

(3) $\left\langle\operatorname{Res}_{H}^{C(u)} \zeta_{n}, \lambda\right\rangle \in \mathbb{Q}$ for each cyclic $H \subseteq C(u)$ and $\lambda \in I(H)$; and

(4) $\zeta_{n}$ is constant on rational conjugacy classes of $C(u)$.

Proof. The equivalence of (1) and (2) is an immediate consequence of (2.4). Moreover, if $\zeta_{n} \in R(C(u))$ then $\operatorname{Res}_{H}^{C(u)} \zeta_{n} \in R(H)$ for any subgroup $H \subseteq$ $C(u)$, and therefore $\left\langle\operatorname{Res}_{H}^{C(u)} \zeta_{n}, \lambda\right\rangle \in \mathbb{Z}$ for $\lambda \in I(H)$. In particular, (2) $\Rightarrow(3)$.

Let $\eta \in I(C(u))$. By Artin's theorem, there are cyclic subgroups $H_{i, \eta} \subseteq$ $C(u), \lambda_{i, \eta} \in I\left(H_{i, \eta}\right)$ and $c_{i, \eta} \in \mathbb{Q}$ such that $\eta=\sum_{i} c_{i, \eta} \operatorname{Ind}_{H_{i, \eta}}^{C(u)}\left(\lambda_{i, \eta}\right)$. Using $(2.2)$, we have

$$
\begin{aligned}
\nu_{n}\left(\chi_{\eta}\right)=\left\langle\zeta_{n}, \eta\right\rangle_{C(u)} & =\sum_{i} c_{i, \eta}\left\langle\zeta_{n}, \operatorname{Ind}_{H_{i, \eta}}^{C(u)}\left(\lambda_{i, \eta}\right)\right\rangle_{C(u)} \\
& =\sum_{i} c_{i, \eta}\left\langle\operatorname{Res}_{H_{i, \eta}}^{C(u)}\left(\zeta_{n}\right), \lambda_{i, \eta}\right\rangle_{H_{i, \eta}}
\end{aligned}
$$

where the third equality uses Frobenius Reciprocity [S, 7.2]. Since $\nu_{n}\left(\chi_{\eta}\right)$ is an algebraic integer, it follows that a sufficient condition for $\nu_{n}\left(\chi_{\eta}\right) \in \mathbb{Z}$ is that for each $i$ we have $\left\langle\operatorname{Res}_{H_{i, \eta}}^{C(u)}\left(\zeta_{n}\right), \lambda_{i, \eta}\right\rangle_{H_{i, \eta}} \in \mathbb{Q}$. Hence, $(3) \Rightarrow(1)$. 
We have seen that $\zeta_{n}$ is a rational-valued class function on $C(u)$. Then it is well-known ([S], Proposition 34 and Theorem 29) that (4) holds if, and only if, $\zeta_{n}$ is a $\mathbb{Q}$-linear combination of (rational) characters of $C(u)$. This in turn is equivalent to the statement $\nu_{n}\left(\chi_{\eta}\right) \in \mathbb{Q}$ for $\eta \in I(C(u))$ thanks to $(2.4)$, and because each $\nu_{n}\left(\chi_{\eta}\right)$ is an algebraic integer, this is equivalent to $(1)$. The theorem is proved.

We restate Theorem 3.1(4) more explicitly, as it is fundamental for later results.

Corollary 3.2. Let $n$ be a positive integer. Then $G$ is an $F S Z_{n}$-group if, and only if, for all commuting pairs of elements $u, g$ and all integers $m$ with $\operatorname{gcd}(m,|G|)=1$, we have

$$
\left|G_{n}(u, g)\right|=\left|G_{n}\left(u, g^{m}\right)\right|
$$

We give two immediate applications of Theorem 3.1.

Lemma 3.4. Let $n$ be a positive integer, and suppose that $G_{n}(u)$ is closed with respect to all power maps $a \mapsto a^{m}$, for $\operatorname{gcd}(m,|G|)=1$. Then (1)-(4) of Theorem 3.1 hold.

Proof. Using (2.2), the hypotheses of the Lemma imply that $\nu_{n}(\eta)$ is invariant under all Galois automorphisms of the cyclotomic field of order $|G|$ for each $\eta \in I(C(u))$. It is therefore rational, and hence integral, as required. Alternatively, the assumptions of the Theorem state that $a^{n}=\left(a u^{-1}\right)^{n}$ implies $a^{m n}=\left(a^{m} u^{-1}\right)^{n}$ whenever $(m,|G|)=1$. Then (3.3) holds because the map $x \mapsto x^{m}$ is bijective on $G$ whenever $\operatorname{gcd}(m,|G|)=1$.

Lemma 3.5. Let $n$ be a positive integer, and suppose that the centralizer of every element of $G$ of order not 1,2,3,4 or 6 is $F S Z_{n}$. Then $G$ is $F S Z_{n}$.

Proof. We show that (3.3) holds for every pair of commuting elements $u, g \in$ $G$. Suppose first that $g$ has order $1,2,3,4$ or 6 . Then $g^{m}=g^{ \pm 1}$, and $(3.3)$ follows from Lemma 2.7. Now suppose that the order of $g$ is 5 or at least 7 . If $\operatorname{gcd}(m,|G|)=1$ then $u \in C(g)$ and $G_{n}(u, g), G_{n}\left(u, g^{m}\right) \subseteq C(g)$. Therefore, $\left|G_{n}(u, g)\right|=\left|G_{n}\left(u, g^{m}\right)\right|$ by Corollary 3.2 because $C(g)$ is an $F S Z_{n}$-group by hypothesis. This proves the lemma.

To facilitate further applications of Corollary 3.2, we introduce the idea of $F S Z^{+}$-groups. To this end, notice that the subgroup $C_{G}(u, g) \subseteq G$ that 
commutes with both $u$ and $g$ acts by conjugation on $G_{n}\left(u, g^{m}\right)$. We can thus consider two properties that qualitatively strengthen (3.3):

$$
\begin{aligned}
& G_{n}(u, g) \text { and } G_{n}\left(u, g^{m}\right) \text { are isomorphic } C_{G}(u, g) \text {-sets. } \\
& G_{n}(u, g) \text { and } G_{n}\left(u, g^{m}\right) \text { afford isomorphic permutation modules } \\
& \quad \text { for } C_{G}(u, g) \text {. }
\end{aligned}
$$

Remark. It is well known that (3.7) is a weaker condition than (3.6). That is, for a group $H$, isomorphic $H$-sets afford isomorphic permutation modules for $H$; but the converse is generally false.

Definition 3.7. $G$ is called an $F S Z_{n}^{+}$-group if (3.7) holds for all commuting pairs of elements $u, g \in G$ and all integers $m$ with $\operatorname{gcd}(m,|G|)=1$, and $G$ is called an $F S Z^{+}$-group if it is $F S Z_{n}^{+}$for all $n$.

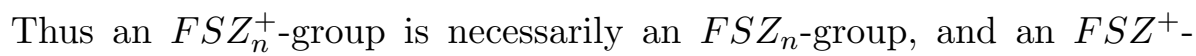
group is necessarily an $F S Z$-group.

Theorem 3.8. Let $n$ be a positive integer. The following are equivalent:

(a) $G$ is an $F S Z_{n}^{+}$-group;

(b) The centralizer of every non-identity element in $G$ is an $F S Z_{n}$-group.

Proof. Condition (3.7) means that each $h \in C(u, g)$ leaves invariant (i.e., commutes with) the same number of elements in both $G_{n}(u, g)$ and $G_{n}(u$, $\left.g^{m}\right)$. Thus, we have

$$
\begin{aligned}
G \text { is } F S Z_{n}^{+} \Leftrightarrow & \left|G_{n}(u, g) \cap C(h)\right|=\left|G_{n}\left(u, g^{m}\right) \cap C(h)\right| \\
& (h \in C(u, g), u g=g u) \\
\Leftrightarrow & \left|G_{n}^{C(h)}(u, g)\right|=\left|G_{n}^{C(h)}\left(u, g^{m}\right)\right|(\langle h, u, g\rangle \text { abelian }) \\
\Leftrightarrow & \left|G_{n}^{C(h)}(u, g)\right|=\left|G_{n}^{C(h)}\left(u, g^{m}\right)\right|(u, g \in C(h), u g=g u) \\
\Leftrightarrow & C(h) \text { is } F S Z_{n}(h \in G) \\
\Leftrightarrow & C(h) \text { is } F S Z_{n}(1 \neq h \in G) .
\end{aligned}
$$

Here, we applied Corollary 3.2 (with $G$ replaced by $C(h)$ ) to get the penultimate equivalence, and Lemma 3.5 to get the last equivalence. 


\section{Some classes of $F S Z$-groups}

We start by applying Theorem 3.8 to show that various families of groups $G$ are $F S Z^{+}$.

Example $4.1\left(\boldsymbol{P S L}_{\mathbf{2}}(\boldsymbol{q})\right)$. Abelian groups are obviously $F S Z$, and dihedral groups are too $[\mathrm{K}]$. Because $P S L_{2}(q)(q \geq 2$ a prime power) has the property that every non-identity element has an abelian or dihedral centralizer, we can conclude that $P S L_{2}(q)$ is an $F S Z^{+}$-group. $\left(P S L_{2}(q)\right.$ simple if $q \geq 4$.)

Example 4.2 ( $C A$-groups). $G$ is a $C A$-group if the centralizer of every non-identity element is abelian. By Theorem 3.8, every $C A$-group is $F S Z^{+}$. With more effort, we can give explicit formulas for, and interpretations of, the indicators in this case.

First we remark that if $g \neq 1$, then $G_{n}(u, g)=\left\{a \in C(u) \mid a^{n}=g\right\}$, and if $G_{n}(u, g) \neq \phi$, then $u^{n}=1$. This holds since if $a \in G_{n}(u, g)$, then $a u^{-1}$ and $a$ commute with $g$, hence with each other. Thus $a \in C(u),\left(a u^{-1}\right)^{n}=$ $a^{n} u^{-n}=a^{n}$, and $u^{n}=1$.

Case 1. $u^{n} \neq 1$. In this case, the remark shows that $G_{n}(u, g)=\phi$ if $g \neq 1$. Thus, $\zeta_{n}(g)=0$ for $g \neq 1$, and of course we also have $\zeta_{n}(1)=\left|G_{n}(u, 1)\right|$. Next, $C(u)$ acts by conjugation on $G_{n}(u, 1)$, and by definition the permutation character $\pi$ of $C(u)$ furnished by $G_{n}(u, 1)$ satisfies $\pi(g)=\mid\{x \in$ $\left.G_{n}(u, 1) \mid g x g^{-1}=x\right\} \mid$ for $g \in C(u)$.

We assert that $\zeta_{n}=\pi$. Certainly $\pi(1)=\left|G_{n}(u, 1)\right|$. Furthermore, if $1 \neq$ $g \in C(u)$ and $x \in G_{n}(u, 1)$, then $x$ and $u$ both commute with $g$, hence with each other. But then $\left(x u^{-1}\right)^{n}=x^{n}$ implies that $u^{n}=1$, contradiction. So there is no such $x$, whence $\pi(g)=0$ for $g \neq 1$. Having shown that $\zeta_{n}(g)=$ $\pi(g)$ for all $g \in C(u)$, our assertion is proved.

By a standard (and straightforward) result, a character $\mu$ of $C(u)$ that vanishes away from the identity element is necessarily a multiple of the regular representation $\rho_{C(u)}$ of $C(u)$, and the multiplicity is $\mu(1) /|C(u)|$. Since this applies with $\mu=\zeta_{n}$, we have $\zeta_{n}=(\zeta(1) /|C(u)|) \rho_{C(u)}$. But the decomposition of the regular representation into irreducibles is standard, namely $\rho_{C(u)}=\sum_{\eta \in I(C(u))} \eta(1) \eta$. Putting all of this together, we find that

$$
\zeta_{n}=\frac{\left|G_{n}(u, 1)\right|}{|C(u)|} \sum_{\eta \in I(C(u))} \eta(1) \eta .
$$


Then comparison with (2.4) shows that

$$
\nu_{n}\left(\chi_{\eta}\right)=\frac{\left|G_{n}(u, 1)\right| \eta(1)}{|C(u)|}
$$

So either the indicators $\nu_{n}\left(\chi_{\eta}\right)$ are all positive, or else $G_{n}(u, 1)=\phi$ and they are all 0 .

Case 2. $u^{n}=1$. In this case, $C(u) \subseteq G_{n}(u)$, but once this is taken into account the arguments of Case 1 carry over. Thus by the previous remark, $\zeta_{n}(g)=\left|\left\{a \in C(u) \mid a^{n}=g\right\}\right|$ when $g \neq 1$. Comparing this with (2.6), we see that $\zeta_{n}$ and $\varphi_{n}^{C(u)}$ agree away from the identity, so that $\zeta_{n}-\varphi_{n}^{C(u)}$ is a multiple of $\rho_{C(u)}$. The multiple in question is

$$
\left(\zeta_{n}(1)-\varphi_{n}^{C(u)}(1)\right) /|C(u)|=\left(\left|G_{n}(u, 1)\right|-\left|\left\{a \in C(u) \mid a^{n}=1\right\}\right|\right) /|C(u)|,
$$

the number of orbits of the conjugation action of $C(u)$ on $G_{n}(u, 1) \backslash$ $\left\{a \in C(u) \mid a^{n}=1\right\}$. As before, this leads to

$$
\zeta_{n}-\varphi_{n}^{C(u)}=\frac{\left|G_{n}(u, 1)\right|-\left|\left\{a \in C(u) \mid a^{n}=1\right\}\right|}{|C(u)|} \sum_{\eta \in I(C(u))} \eta(1) \eta
$$

and

$$
\nu_{n}\left(\chi_{\eta}\right)=\nu_{n}^{C}(\eta)+\frac{\eta(1)\left(\left|G_{n}(u, 1)\right|-\left|\left\{a \in C(u) \mid a^{n}=1\right\}\right|\right)}{|C(u)|} .
$$

Example 4.3 (Regular $p$-groups). Fix a prime $p$. For an introduction to regular $p$-groups, see [H, Chapter 12.4]. All we need to know is that in a regular $p$-group, the equation $a^{k}=b^{k}$ holds if, and only if, $\left(a b^{-1}\right)^{k}=1$ for $k \in \mathbb{Z}$ $[\mathrm{H}$, Theorem 12.4.4]. We show that a regular $p$-group $G$ is $F S Z^{+}$.

If $a \in G$ then $\left(a u^{-1}\right)^{n}=a^{n} \Leftrightarrow\left(a u^{-1} a^{-1}\right)^{n}=1 \Leftrightarrow u^{n}=1$. This shows that either $u^{n} \neq 1$ and $G_{n}(u)=\phi$; or $u^{n}=1$ and $G_{n}(u)=G$. Now Lemma 3.4 applies, and shows that $G$ is $F S Z$. But every subgroup of a regular $p$-group is also regular (loc. cit.), so Theorem 3.8 now tells us that $G$ is $F S Z^{+}$. We can again make the indicators more explicit. If $G_{n}(u)=\phi$ then $\zeta_{n}$ vanishes identically and the corresponding indicators $\nu_{n}\left(\chi_{\eta}\right)=0$. On 
the other hand, if $u^{n}=1$ then $G_{n}(u, g)=\left\{a \in G \mid a^{n}=g\right\}$. Since $\zeta_{n}(g)=$ $\left|G_{n}(u, g)\right|$, this means that for $g \in C(u)$ the values of $\zeta_{n}$ coincide with those of the generalized character $\varphi_{n}^{G}=\sum_{\eta} \nu_{n}^{G}(\eta) \eta(2.6)$, i.e.,

$$
\zeta_{n}=\operatorname{Res}_{C(u)}^{G} \varphi_{n}^{G}
$$

We note that all $p$-groups of order less than $p^{p+1}$, nilpotence class less than $p$, or exponent $p$, are regular $[\mathrm{H}]$, and therefore FSZ. We will see later that there are groups of order $5^{6}$ and exponent $5^{2}$ which are not FSZ.

Example 4.4 (Wreathed products $Z_{p} \prec Z_{p}$ ). This $p$-group (the regular wreathed product of $Z_{p}$ with itself), is an irregular $p$-group of order $p^{p+1}$. We will show that it is $F S Z^{+}$, so that the class of $F S Z$ p-groups is strictly larger than the class of regular $p$-groups.

$G$ has a normal elementary abelian $p$-subgroup $V$ of order $p^{p}$ (which we consider as a $G F(p)$-vector space of dimension $p$ ) and a cyclic subgroup $J=\langle j\rangle$ of order $p$ such that $j$ cyclically permutes a basis of $V$. We identify $j$ with the $p \times p$ Jordan block

$$
j=\left(\begin{array}{llll}
0 & & & 1 \\
1 & & & \\
& \ddots & & \\
& & 1 & 0
\end{array}\right) .
$$

Then $G=J \ltimes V$, and multiplication in $G$ is $\left(j^{r}, x\right)\left(j^{s}, y\right)=\left(j^{r+s}, j^{s} x+y\right)$. The subgroup of $G$ generated by $p$ th powers coincides with the center $Z:=$ $Z(G) . Z \subseteq V$ is 1 -dimensional and spanned by the all 1's vector 1 . The operator $k:=1+j+\cdots+j^{p-1}$ plays a rôle below; $k$ is the all 1 s matrix and $k V=Z$.

Because $G$ has exponent $p^{2}$, in proving that $G$ is $F S Z$ it suffices to consider only the cases $n=p, p^{2}$, and of these only $n=p$ is in doubt. Fix some $u^{-1}:=\left(j^{d}, t\right) \in G$. By what we have said, $G_{p}(u, g)=\phi$ unless $g \in Z$. We show that (3.3) holds with $n=p$ and $1 \neq g \in Z$.

An element $a=\left(j^{i}, v\right)$ satisfies $a^{p}=(1, k v)$ if $j^{i} \neq 1$, and otherwise $a^{p}=$ 1. Moreover, $a u^{-1}=\left(j^{i+d}, j^{d} v+t\right)$. Therefore, $\left(a u^{-1}\right)^{p}=a^{p}=g$ if, and only if, the four conditions $i \not \equiv 0(\bmod p), i+d \not \equiv 0(\bmod p), k v=g, k\left(j^{d} v+t\right)=g$ all hold. But $k\left(j^{d} v+t\right)=k v+k t$ (because $k$ is the all 1 s matrix and $j^{d}$ 
a permutation matrix). So if the four conditions hold then we must have $k t=0$. Turned around, if $u^{-1}=\left(j^{d}, t\right)$ and $k t \neq 0$ then $G_{p}(u, g)=\phi$ for any non-zero $g \in Z$. If, on the other hand, $k t=0$, then $G_{p}(u, g)=\left\{\left(j^{i}, v\right) \mid i \not \equiv\right.$ $0,-d(\bmod p), k v=g\}$ has cardinality $(p-1) p^{p-1}$ or $(p-2) p^{p-1}$ depending on whether or not $d \equiv 0(\bmod p)$. So in all cases, the desired property of $G_{p}(u, g)$ is established. Note that the hypotheses of Lemma 3.4 do not hold in this case, so they are sufficient but not necessary.

Example 4.5 (Direct products). It follows easily from the definition that, in an obvious sense, $\left|G_{n}(u, g)\right|$ is multiplicative over direct products of groups. Thus the class of FSZ groups is closed under direct products; for example, a nilpotent group, all of whose Sylow subgroups are regular, is $F S Z$. On the other hand, the direct product of two groups, one of which is $F S Z$ and one which is not, is itself not FSZ. So once we have found a non- $F S Z$ group $F$, say, there are large numbers of non- $F S Z$ groups having $F$ as a direct factor.

Example 4.6 (Regular CN-groups). $G$ is a $C N$-group if the centralizer of every non-identity element is nilpotent (i.e., a direct product of $p$-groups). We call $G$ a regular CN-group if the centralizer of every non-identity element is a direct product of regular p-groups. By Theorem 3.8 together with Examples 4.3 and 4.5 above, every regular CN-group is FSZ.

Example 4.7 (Mathieu group $M_{11}$ ). We show that $M_{11}$ is $F S Z$ by applying Lemma 3.5. Indeed, it is readily checked [AT] that centralizers of elements of order 5 or greater than 6 are $F S Z$, and the result follows.

\section{A sufficiency condition and groups of special orders}

We start by showing that (3.6) holds automatically for some values of $m$.

Lemma 5.1. Suppose that $m \in \mathbb{Z}$ satisfies $\operatorname{gcd}(m,|G|)=1$ and $m \equiv \pm 1$ $(\bmod n)$. Then for all pairs of commuting elements $u, g \in G$, there is an isomorphism of $C(u, g)$-sets

$$
G_{n}(u, g) \rightarrow G_{n}\left(u, g^{m}\right), \quad a \mapsto a^{m} .
$$


Proof. Let $a \in G_{n}(u, g)$, so that $\left(a u^{-1}\right)^{n}=a^{n}=g$, and suppose first that $m \equiv 1(\bmod n)$. Then

$$
\begin{aligned}
\left(a^{m} u^{-1}\right)^{n} & =\left(a^{m-1} a u^{-1}\right)^{n} \\
& \left.=a^{(m-1) n}\left(a u^{-1}\right)^{n} \quad \text { (because } a^{m-1} \in\left\langle a^{n}\right\rangle \subseteq C(u)\right) \\
& =a^{m n}
\end{aligned}
$$

This argument shows that the $m$ th power map $\varphi_{m}: a \mapsto a^{m}$ induces an injection $G_{n}(u, g) \rightarrow G_{n}\left(u, g^{m}\right)$. If $m^{\prime}$ is the inverse of $m(\bmod n)$, then $\varphi_{m^{\prime}}$ is the inverse of $\varphi_{m}$, so that $\varphi_{m}$ is a bijection. Moreover, because conjugation commutes with power maps, $\varphi_{m}$ is also a morphism of $C(u, g)$-sets.

This completes the proof of the lemma when $m \equiv 1(\bmod n)$. If $m \equiv$ $-1(\bmod n)$, the result follows from the case $m \equiv 1(\bmod n)$ together with Lemma 2.7, because the bijection of Lemma 2.7 is $C(u, g)$-equivariant.

For some groups, Lemma 5.1 tells us all we need to know in order to deduce the $F S Z_{n}^{+}$property. This is the essential content of

Theorem 5.2. Suppose that for each element $a \in G$ we have

$$
o\left(a^{n}\right) / o\left(a^{n^{2}}\right) \in\{1,2,3,4,6\} .
$$

Then $G$ is an $F S Z_{n}^{+}$-group.

Proof. It suffices to show that (3.6) holds for all commuting pairs of elements $u, g$ and integers $m$ coprime to $|G|$. For in this case, the Remark that follows (3.7) shows that $G$ is $F S Z_{n}^{+}$.

Suppose that $o(g)=t$. If $G_{n}\left(u, g^{m}\right)=\phi$ for all $m$ there is nothing to prove, so assume that $a \in G_{n}\left(u, g^{m}\right)$ for some $m$. Thus $a^{n}=g^{m}$ has order $t$, and $a^{n^{2}}=g^{m n}$ has order $t / g c d(t, n)$. Thus $o\left(a^{n}\right) / o\left(a^{n^{2}}\right)=\operatorname{gcd}(t, n)$. As a result, the hypothesis of the Theorem tells us that

$$
\operatorname{gcd}(t, n) \in\{1,2,3,4,6\}
$$

Set $d=\operatorname{gcd}(t, n)$. Because $m$ is coprime to $t$ (Cauchy's theorem) it is also coprime to $d$. Because of (5.2), it follows that

$$
m \equiv \pm 1(\bmod d)
$$

There are integers $a, b$ such that $a t+b n=d$. Then for some integer $k$ we have $m= \pm 1+k d= \pm 1+k a t+k b n$ and therefore $g^{m}=g^{ \pm 1+k b n}$. Now by 
Lemma 5.1 we see that there is an isomorphism of $C(u, g)$-sets $G_{n}(u, g) \rightarrow$ $G_{n}\left(u, g^{m}\right)$, as required. This completes the proof of the theorem.

The theorem has some interesting consequences.

Corollary 5.3. Suppose that the order of each element of $G$ divides $2^{\alpha} 3^{\beta} q$, where $q$ is a squarefree integer coprime to 6 and either $\alpha, \beta \leq 3$ or $\beta \leq 1$ and $\alpha \leq 5$. Then $G$ is $F S Z^{+}$.

Proof. The hypothesis of the previous theorem hold for all $n$.

Corollary 5.4. Suppose that $n \in\{1,2,3,4,6\}$. Then every group $G$ is $F S Z_{n}^{+}$.

Proof. We always have $o\left(a^{n}\right) / o\left(a^{n^{2}}\right) \mid n$ for $a \in G$ (see the proof of Theorem 5.2).

Corollary 5.5. All groups of order less than 100 are $F S Z^{+}$.

Apropos of our introductory comments that some of our stated results are not best-possible, this is certainly true of the preceding Corollary. Because all groups of order $512=2^{9}$ are FSZ (Section 7), one could check all groups up to order $728=3^{6}-1$ rather easily. It is very likely that the non-FSZ-groups of least order are irregular $p$-groups of order $2^{k}(10 \leq k \leq$ 13), $3^{k}(6 \leq k \leq 8)$, or $5^{6}$.

Corollary 5.6. The Mathieu groups $M_{11}, M_{12}$ are $F S Z^{+}$.

Proof. We have seen before that the Mathieu Group $M_{11}$ is FSZ. Alternatively, the exponent of both $M_{11}$ and $M_{12}$ is $2^{3} \cdot 3 \cdot 5 \cdot 11$. Thus we may apply Corollary 5.3 to see that, in fact, both $M_{11}$ and $M_{12}$ are $F S Z^{+}$.

\section{Symmetric groups}

The next theorem was proved independently by the present authors and Pavel Etingof; here we give our proof.

Theorem 6.1. The symmetric group $S_{N}$ is an FSZ-group.

Proof. We have to show that (3.3) holds for all commuting pairs $u, g \in S_{N}$ and all $m$ coprime to $N$ !. There is nothing to prove if $g=1$, so assume that $1 \neq g$ is a product of $e_{i}$ cycles of length $i$. Thus $\sum_{i} i e_{i}=N$, and $C(g)$ is 
contained in a subgroup of $S_{N}$ isomorphic to $S_{e_{1}} \times S_{2 e_{2}} \times \cdots \times S_{N e_{N}}$. Using induction on $N$ and the multiplicativity of $G_{n}(u, g)$ over direct products (Section 4, Example 5), we may, and shall, assume that $g$ is a product of $e$ cycles of length $r$, with $N=e r$. Then $C(g)=S_{e} \iota Z_{r}=S \ltimes V$ where $S=S_{e}$ and $V=Z_{r}^{e}$. We use additive notation for $V$, and write $g=\left(1, \mathrm{~g}_{0}, \ldots, \mathrm{g}_{0}\right)$ with $\left(g_{0}, \ldots, g_{0}\right) \in Z_{r}^{e}$ for some generator $g_{0}$ of $Z_{r}$. In effect, $V$ is a right $Z_{r} S$-module with $S$-action denoted exponentially by $v^{s}$ (which is right conjugation $s^{-1} v s$ in $C(g)$ ), and multiplication in $C(g)$ is

$$
(s, v)(t, w)=\left(s t, v^{t}+w\right) .
$$

Then

$$
(s, v)^{n}=\left(s^{n}, v+v^{s}+\cdots+v^{s^{n-1}}\right) .
$$

Let $(s, v)=s v \in C(g)(s \in S, v \in V)$, and let $s=s_{1} \ldots s_{q}$ be a representation of $s$ as a product of disjoint cycles in $S_{e}$. There is a corresponding decomposition of $v=v_{1}+\cdots+v_{q}$ such that for each index $i, s_{i}$ has length $f_{i}, v_{i} \in W_{i} \cong Z_{r}^{f_{i}}$, and $s v=\left(s_{1} v_{1}\right) \ldots\left(s_{q} v_{q}\right) \in\left(S_{f_{1}} \prec Z_{r}\right) \times \cdots \times\left(S_{f_{q}} \prec Z_{r}\right)$.

Now suppose that $(s, v)^{n}=g^{m}=(1, m g)=\left(1, m g_{0}, \ldots, m g_{0}\right)$. Then for each index $i$ we have $\left(s_{i}, v_{i}\right)^{n}=\left(1, m g_{0}, \ldots m g_{0}\right) \in S_{f_{i}}<Z_{r}$. Then $s_{i}^{n}=1$, whence $f_{i} \mid n$; moreover $\left(m g_{0}, \ldots, m g_{0}\right)=v_{i}+v_{i}^{s_{i}}+\cdots+v_{i}^{s_{i}^{n-1}}=\left(n / f_{i}\right)\left(v_{i}+\right.$ $\left.v_{i}^{s_{i}}+\cdots+v_{i}^{s_{i}^{f_{i}-1}}\right)$. We can write this more compactly as

$$
m\left(g_{0}, \ldots, g_{0}\right)=\left(n / f_{i}\right) N_{s_{i}}\left(v_{i}\right) \in Z_{r}^{f_{i}}
$$

where $N_{s_{i}}$ denotes norm with respect to $\left\langle s_{i}\right\rangle$. Note that because the left-hand side of (6.1) has order exactly $r$ (as an element of $V$ ), then $\operatorname{gcd}\left(n / f_{i}, r\right)=1$. Setting $l=\operatorname{gcd}(n, r)$, it follows that the condition $l \mid f_{i}$ (for all indices $i$ ) is necessary in order that there be any solutions of the equation $(s, v)^{n}=g^{m}$.

It is also sufficient: if $\operatorname{gcd}\left(n / f_{i}, r\right)=1$, we can always find $v_{i}^{\prime} \in W_{i}:=$ $Z_{r}^{f_{i}}$ whose norm is $\left(n / f_{i}\right)^{-1} m\left(g_{0}, \ldots, g_{0}\right)$. The element $v_{i}^{\prime \prime}=\left(g_{0}, 0, \ldots, 0, g_{0}\right.$, $\left.0, \ldots, 0, \ldots, g_{0}, \ldots, 0\right)$, of length $f_{i}$ and with $g_{0}$ 's occurring after $l-1$ zeros, has norm $\left(f_{i} / l\right)\left(g_{0}, \ldots, g_{0}\right)$, and we take $v_{i}^{\prime}=m(n / l)^{-1}\left(v_{i}^{\prime \prime}\right)^{s}$. Here inverses are taken modulo $r$.

The set of all $v_{i} \in W_{i}$ satisfying (6.1) is

$$
v_{i}^{\prime}+\operatorname{ker} N_{s_{i}}=v_{i}^{\prime}+\left[W_{i}, s_{i}\right]
$$

where $\left[W_{i}, s_{i}\right] \subseteq W_{i}$ is spanned by $w-w^{s_{i}}, w \in W_{i}$. Because $s_{j}$ acts trivially on $W_{i}$ for $i \neq j$, we have $\left[W_{i}, s_{i}\right]=\left[W_{i}, s\right]$. It follows that, for fixed $s \in S$ as 
above, the set of all $v \in V$ satisfying $(s, v)^{n}=g^{m}$ is

$$
v_{1}^{\prime}+\cdots+v_{q}^{\prime}+[V, s] .
$$

Fix a second element $(t, w) \in C(g)$, so that $(s, v)(t, w)=\left(s t, v^{t}+w\right)$. By the foregoing, the set of $x \in V$ such that $(s t, x)^{n}=g^{m}$ is the coset

$$
x_{1}^{\prime}+\cdots+x_{p}^{\prime}+[V, s t]
$$

where $s t$ is a product of $p$ disjoint cycles of lengths $k_{1}, \ldots, k_{p}$ and $x_{i}^{\prime}=$ $m(n / l)^{-1}\left(x_{i}^{\prime \prime}\right)^{s t}$ with $x_{i}^{\prime \prime}=\left(g_{0}, 0, \ldots, 0, g_{0}, 0, \ldots, 0, g_{0}, \ldots 0\right)$ similarly to the previous case. Hence, for fixed $s \in S$ and fixed $(t, w) \in C(g)$, the cardinality of the set of elements $v \in V$ such that $(s, v)^{n}=((s, v)(t, w))^{n}=g^{m}$ is

$$
\begin{aligned}
\left|\left(x_{1}^{\prime}+\cdots+x_{p}^{\prime}+[V, s t]\right) \cap\left(w+\left(v_{1}^{\prime}+\cdots+v_{q}^{\prime}+[V, s]\right)^{t}\right)\right| \\
=\mid m(n / l)^{-1}\left\{\left(\sum_{i=1}^{p}\left(x_{i}^{\prime \prime}\right)^{s t}+[V, s t]\right)\right. \\
\left.\quad \cap\left(m^{-1}(n / l) w^{t^{-1}}+\sum_{i=1}^{q}\left(v_{i}^{\prime \prime}\right)^{s}+[V, s]\right)^{t}\right\} \mid .
\end{aligned}
$$

Now we have gone out of our way to arrange that $\sum_{i} x_{i}^{\prime \prime}=\sum_{i} v_{i}^{\prime \prime}$. Because $s$ and $s t$ leave invariant $[V, s]$ and $[V, s t]$, respectively, it follows that the cardinality of the last set is

$$
\begin{aligned}
& \left|\left(\sum_{i=1}^{p} x_{i}^{\prime \prime}+[V, s t]\right)^{s t} \cap\left(m^{-1}(n / l) w^{(s t)^{-1}}+\sum_{i=1}^{q} v_{i}^{\prime \prime}+[V, s]\right)^{s t}\right| \\
& \quad=\left|([V, s t]) \cap\left(m^{-1}(n / l) w^{(s t)^{-1}}+[V, s]\right)\right|
\end{aligned}
$$

This equals 0 if $m^{-1}(n / l) w^{(s t)^{-1}} \notin[V, s t]+[V, s]$, and equals $|[V, s t] \cap[V, s]|$ if $m^{-1}(n / l) w^{(s t)^{-1}} \in[V, s t]+[V, s]$, and in both cases the condition is independent of $m$ coprime to $r$. This completes the proof of the theorem.

The proof of the theorem also yields

Corollary 6.2. The alternating group $A_{N}$ is an FSZ-group.

Proof. Keep the same notation as before, now with $g \in A_{N}$. Then $C_{A_{N}}(g)=$ $C_{S_{N}}(g) \cong S_{e}$ 乙 $Z_{r}$ if $r$ is even, and $C_{A_{N}}(g) \cong A_{e} \succ Z_{r}$ if $r$ is odd. In either case, 
the proof of the theorem carries over verbatim, because once $s, t, w$ are fixed the rest of the calculation takes place in $V \subseteq A_{N}$.

Somewhat later Etingof proved [E] a natural extension of Theorem 6.1, namely

Theorem 6.3 (Etingof). $S_{N} \succ A$ is an FSZ-group for any finite abelian group $A$.

The proof, which we omit, runs along similar lines to that of Theorem 6.1 , but is more elaborate. As an application, recall that every centralizer in $S_{N}$ is a direct product of wreathed products of the type occurring in Theorem 6.3. Then by Theorem 3.8 we deduce

Corollary 6.4. $S_{N}$ is an $F S Z^{+}{ }_{-}$group.

\section{Counterexamples}

In this section, we consider groups of order $5^{6}=15,625$. We present two GAP programs, one optimized for testing these 5-groups, and the second which is optimized to be used to test the FSZ property any arbitrary group $G$.

Using Corollary 3.2, the first [GAP] program can be used to test integrality of indicators for doubles of $p$-groups of exponent $p^{2}$. On a dual core $3.33 \mathrm{Mz}$ computer with $8 \mathrm{~Gb}$ of Ram, after approximately $6 \mathrm{~h}$ of running for the 555 groups of exponent 25 among the 684 groups of order $15,625=5^{6}$, it found 32 groups whose indicators for the Drinfel'd double are not all integers (only indicator $\nu_{5}$ can be non-integer, and all the other groups of order $5^{6}$ can easily be seen to be FSZ). These are the groups SmallGroup $(12625, k)$ (in the small library) for $k$ being the ID numbers 632-635, 637, 638, 640650, 653-655, and 657-668. We note that groups 662, 665 and 668 have few conjugacy classes, many of which give rise to representations of the Drinfeld double with non-integer indicators, and also several groups $G$ among these have a large number of conjugacy classes and still produce representations of the double with non-integer indicators.

One particular such group is $\operatorname{SmallGroup}(15625,668)$. The GAP program takes parameters $G, p, m$ and is designed to test the integrality of the $m$-indicators of representations of $D(G)$ induced from cyclic subgroups of prime order $p$ (only). This is enough to test the FSZ property for a $p$-group 
of exponent $p^{2}$. The command

$$
\text { FSInd(SmallGroup }(15625,668), 5,5)
$$

tests the integrality of indicators $\nu_{5}$ for representations induced from cyclic groups of order 5 and determines those conjugacy classes (that is, their GAP ID) for which there are associated representations of the double which have non-integral indicators. It runs in a few seconds on the computer hardware configuration mentioned above for group SmallGroup $(15625,668)$.

By Lemma 2.9 all indicators are in $\mathbb{Q}\left(\zeta_{5}\right)$, and since they are also real by Remark 2.8, the non-integer indicators are algebraic integers in $\mathbb{Q}(\sqrt{5}) \backslash \mathbb{Q}$. We confirmed this using a GAP program of Rebecca Courter [C], which computes values for the FS-indicators of irreducible representations of the double of SmallGroup(15625,668).

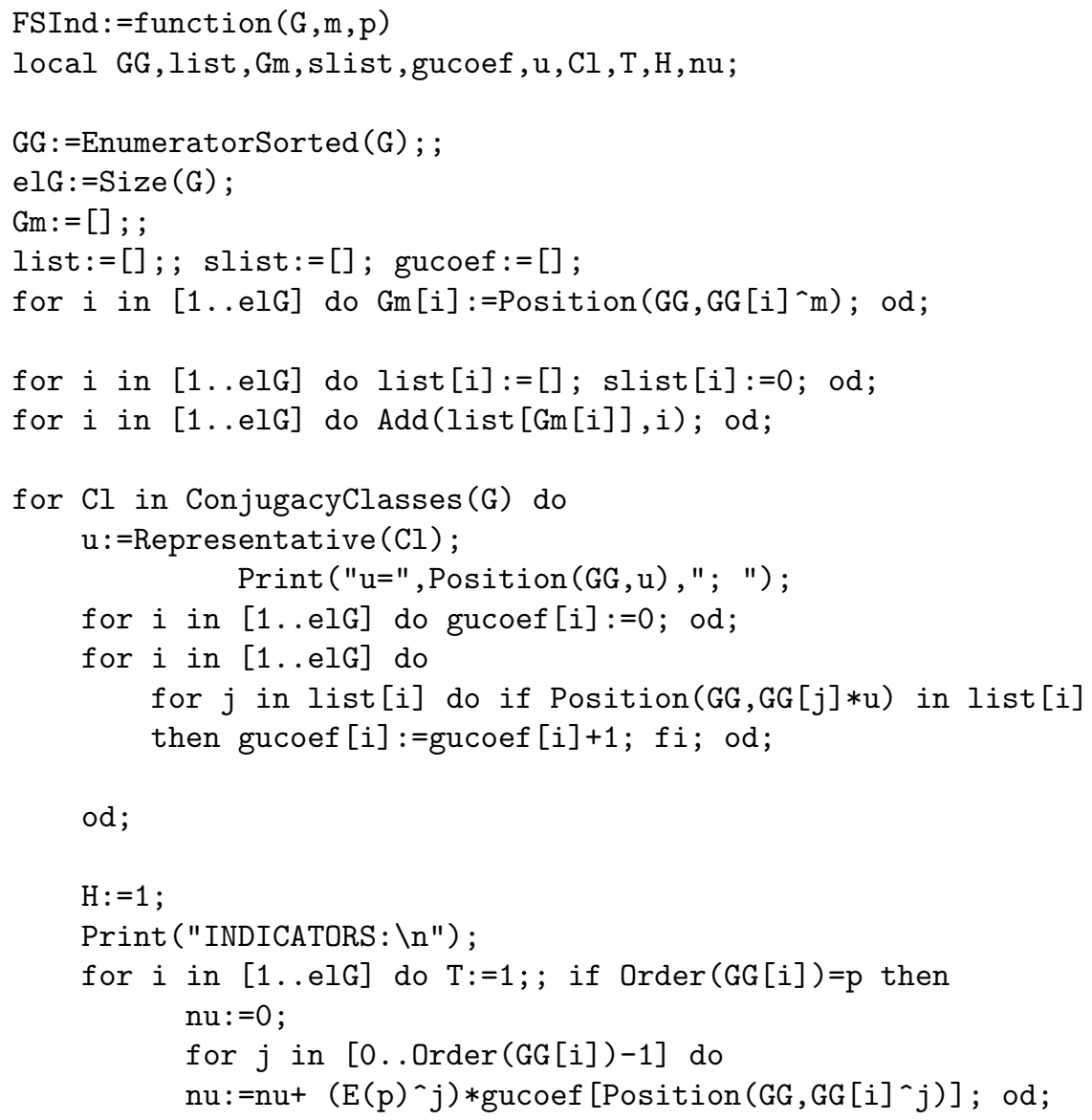




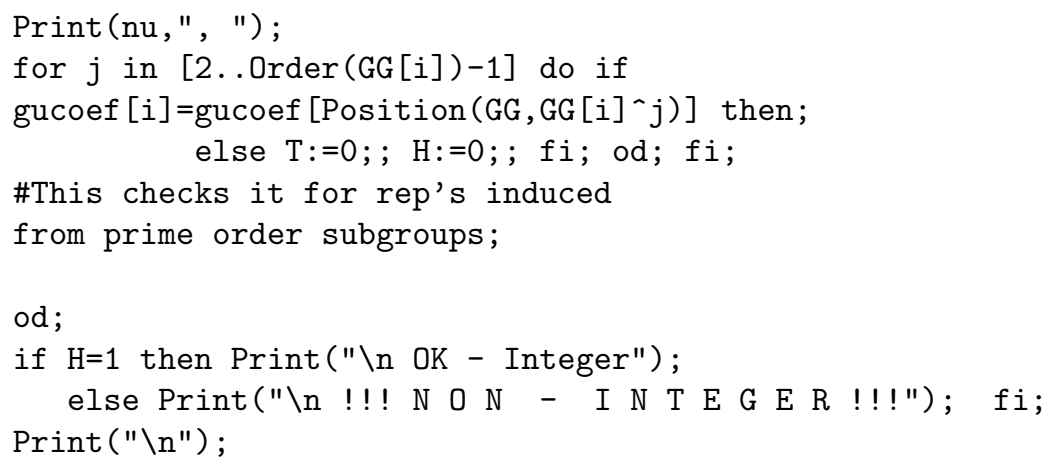

od;

return;

end;

Our second program provides a general computational test for whether the indicators $\nu_{m}$ of all the representations of the double of a group $G$ are integers; it takes the integer $m$ and the group $G$ as parameters. It uses the test provided by Corollary 3.2, and it is optimized to attempt the least possible number of computations of products in $G$ (but uses more memory). This is necessary since products of elements (usually represented as compositions of permutations) tend to take the most time under GAP.

The algorithm runs as follows. The program first creates a list [Gm] with all the $m$ th powers of elements of $G$ (GAP works with list position), and then another variable [list] such that which for each element $x \in G$, list $[\mathrm{x}]$ retains all $h$ for which $h^{m}=x$ (this saves doing computations of the type $\underbrace{h * h * h \cdots * h}$ each time we compute an indicator). Then, we create the list variable [glist], which will retain the sets $\left\{g^{k} \mid \operatorname{gcd}(\operatorname{ord}(g), k)=1\right\}-$ elements generating the same cyclic subgroup, on which we have to test the condition of Corollary 3.2. Next, for each conjugacy class $\mathrm{Cl}$ of $G$, we pick an element of $\mathrm{Cl}$ and we create a list [gucoef] which for each element $g$ of $G$ will count the number of solutions of the equation $h^{m}=(h u)^{m}=g$ (obviously, for the purpose of testing integrality of all indicators, we can just work with $u$ instead of $u^{-1}$ ). This uses the previously created variable [list] for the $m$ th roots of $g$ (so that we don't need to perform supplementary multiplications). Once this is done, using Corollary 3.2, one only needs to check that the number of these solutions is constant on the sets contained in the variable [list]. The printout also shows the GAP ID of some representative $u$ of each 
conjugacy class and whether $C l\left(u^{-1}\right)$ produces some representation of $D(G)$ with non-integer indicator $\nu_{m}$. The program could be slightly optimized to jump out of the loop whenever a non-integer indicator is found, and it can be easily modified to find the indicators for all the representations.

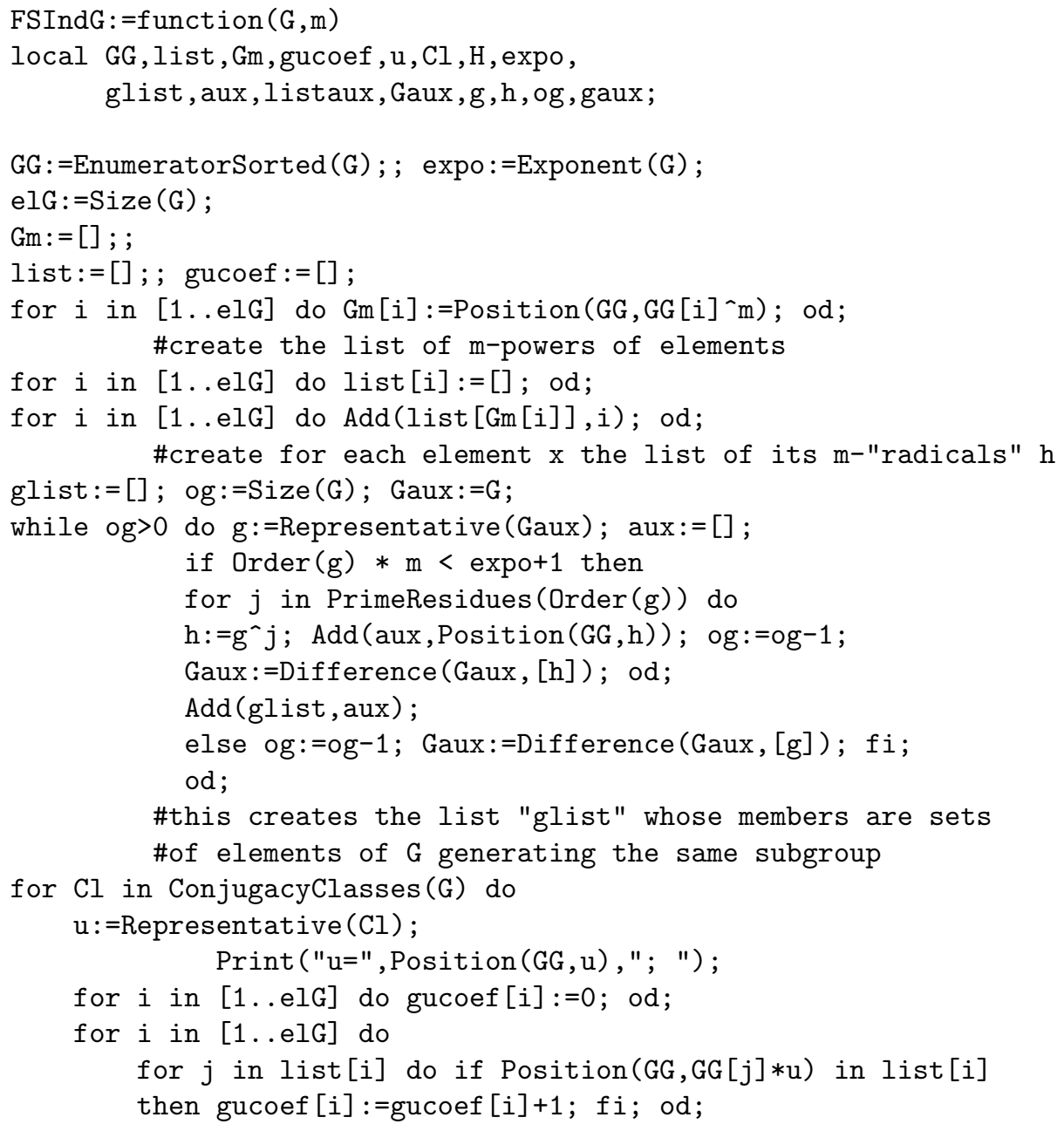


else $H:=0 ;$ fi; od;

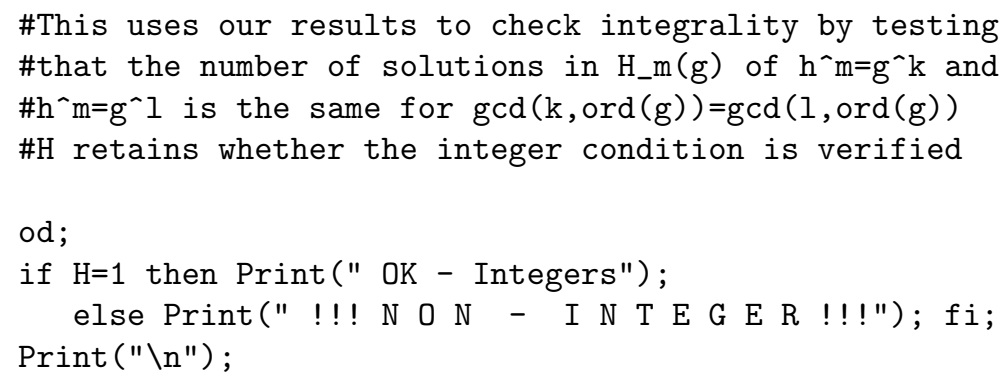

Using this last program, we observed that all the 2-groups up to order 512 are FSZ. Since 2-groups can in general behave differently than other $p$-groups, it is natural to ask whether perhaps all 2-groups are FSZ.

It is also known that all the indicators of the representations of the group $S_{n}$ are non-negative integers [Scf]. Thus one might ask whether this is true for $D\left(S_{n}\right)$ as well. This has been shown in [C] for $n \leq 10$. It is also known that for any dihedral group, all indicators of its double are non-negative $[\mathrm{K}]$.

\section{Acknowledgments}

The first author was supported by an AMS-Simons travel grant and by CNCS grant TE45, no. 88/02.02.2010. The second author was supported by NSF grant DMS-1139676 and the third author by NSF grant DMS-1001547. We thank Siu-Hung Ng and Pavel Etingof for communicating some of their unpublished results to us.

\section{References}

[AT] J.H. Conway et al., Atlas of finite groups, Oxford University Press, 1985.

[C] R. Courter, Computing higher indicators for the double of a symmetric group, Ph.D. thesis, USC, 2012, arXiv:1206.6908.

[CR] C. Curtis and I. Reiner, Methods of representation theory with applications to finite groups and orders, 1, Wiley, New York, 1981. 
[E] P. Etingof, On some properties of quantum doubles of finite groups, J. Algebra 394 (2013), 1-6.

[EG] P. Etingof and S. Gelaki. On the exponent of finite-dimensional Hopf algebras. Math. Res. Lett. 6 (1999), 131-140.

[H] M. Hall Jr., The theory of groups, Macmillian, New York, 1959.

[GAP] The GAP Group, GAP - groups, algorithms, and programming, Version 4.5.5, 2012, available at: http://www.gap-system.org.

[KMM] Y. Kashina, G. Mason and S. Montgomery, Computing the Frobenius-Schur indicator for abelian extensions of Hopf algebras, J. Algebra 251 (2002), 11-24.

[KSZ] Y. Kashina, Y. Sommerhäuser and Y. Zhu, On higher FrobeniusSchur indicators, Mem. Amer. Math. Soc. 181 (2006), no. 855, viii $+65 \mathrm{pp}$.

[K] M. Keilberg, Higher indicators for some groups and their doubles, J Algebra Appl. 11(2) (2012), 38pp.

[LM] V. Linchenko and S. Montgomery, A Frobenius-Schur theorem for Hopf algebras, Algebr. Represent. Theory 3 (2000), $347-355$.

[MN05] G. Mason and S-H. Ng, Central invariants and Frobenius-Schur indicators for semisimple quasi-Hopf algebras, Adv. Math. 190 (2005), 161-195.

[M] S. Montgomery, Hopf algebras and their actions on rings, CBMS Conf. Ser. Math., 82, Amer. Math. Soc., Providence, 1993.

[N] S-H. Ng, private communication, March 2011.

[NS07] S-H. Ng and P. Schauenburg, Frobenius-Schur indicators and exponents of spherical categories, Adv. Math. 211(1) (2007), $34-71$.

[NS11] S-H. Ng and P. Schauenburg, Congruence subgroups and generalized Frobenius-Schur indicators, Comm. Math. Phys. 300(1) (2010), 1-46.

[Scf] T. Scharf, Die Wurzelanzahlfunktion in symmetrischen Gruppen, J. Algebra 139 (1991), 446-457.

[S] J.P. Serre, Linear representations of finite groups, GTM 42, Springer-Verlag, New York-Berlin-Heidelberg, 1977. 
FSZ-groups and Frobenius-Schur indicators of quantum doubles 779

Department of Mathematics

UNIVERSITY OF IOWA AND UNIVERSITY OF BUCHAREST

IOWA City, IA AND Bucharest

ROMANIA

E-mail address: miodrag-iovanov@uiowa.edu, yovanov@gmail.com

Department of Mathematics

University of California at Santa Cruz

Santa Cruz, CA

USA

E-mail address: gem@ucsc.edu

Department of Mathematics

University of SOUTHERn CALIFORNIA

Los Angeles, CA

USA

E-mail address: smontgom@usc.edu

Received August 17, 2012 
